D. Barlet

Nagoya Math. J.

Vol. 171 (2003), 187-196

\title{
REAL CANONICAL CYCLE AND ASYMPTOTICS OF OSCILLATING INTEGRALS
}

\author{
DANIEL BARLET
}

\begin{abstract}
Let $X_{\mathbb{R}} \subset \mathbb{R}^{N}$ a real analytic set such that its complexification $X_{\mathbb{C}} \subset \mathbb{C}^{N}$ is normal with an isolated singularity at 0 . Let $f_{\mathbb{R}}: X_{\mathbb{R}} \rightarrow \mathbb{R}$ a real analytic function such that its complexification $f_{\mathbb{C}}: X_{\mathbb{C}} \rightarrow \mathbb{C}$ has an isolated singularity at 0 in $X_{\mathbb{C}}$. Assuming an orientation given on $X_{\mathbb{R}}^{*}$, to a connected component $A$ of $X_{\mathbb{R}}^{*}$ we associate a compact cycle $\Gamma(A)$ in the Milnor fiber of $f_{\mathbb{C}}$ which determines completely the poles of the meromorphic extension of $\int_{A} f^{\lambda} \square$ or equivalently the asymptotics when $\tau \rightarrow \pm \infty$ of the oscillating integrals $\int_{A} e^{i \tau f} \square$. A topological construction of $\Gamma(A)$ is given. This completes the results of $[\mathrm{BM}]$ paragraph 6 .
\end{abstract}

\section{$\S 0$. Introduction}

Let $X_{\mathbb{C}}$ be a normal complex space of dimension $n+1(n \geq 1)$ having an isolated singularity at 0 , and let $f: X_{\mathbb{C}} \rightarrow \mathbb{C}$ be an holomorphic function on $X_{\mathbb{C}}$ with an isolated singularity at 0 . We shall assume that $\left(X_{\mathbb{C}}, f\right)$ is the complexification of a real analytic function $\left(X_{\mathbb{R}}, f_{\mathbb{R}}\right)$ on a real analytic space $X_{\mathbb{R}}$. In such a situation, we shall consider $A$, non zero, in $H^{0}\left(X_{\mathbb{R}}^{*}, \mathbb{C}\right)$. Assuming that an orientation is given on the smooth real manifolds $X_{\mathbb{R}}^{*}$, we have defined in [BM] a compactly supported cohomology class $\gamma(A) \in$ $H_{c}^{n}(F, \mathbb{C})_{1}$ associated to $A$, where $F$ denotes the complex Milnor's fiber of $f$ on $X_{\mathbb{C}}$ and $H_{c}^{n}(F, \mathbb{C})_{1}$ the spectral part for the eigenvalue 1 of the monodromy acting on $H_{c}^{n}(F, \mathbb{C})$. The definition is the following:

For any $e \in H^{n}(F, \mathbb{C})_{1}$ represented by semi-meromorphic forms on $X_{\mathbb{C}}$, with poles along $f=0, w_{0}, \ldots, w_{k}$, so satisfying the conditions

$$
\text { (A) }\left\{\begin{array}{l}
\text { 1) } d w_{j}=\frac{d f}{f} \wedge w_{j-1} \\
\text { 2) }\left[w_{k} /_{F}\right]=e \in H^{n}(F, \mathbb{C})_{1}
\end{array} \quad \forall j \in[1, k], w_{0}=0\right.
$$

Received June 25, 2001.

Revised September 13, 2002.

2000 Mathematics Subject Classification: 32S40, 14P15. 
we have

$$
I(e, \overline{\gamma(A)})=(2 i \pi)^{-n} \operatorname{Res}\left(\lambda=0, \int_{A} f^{\lambda} \rho w \wedge \frac{d f}{f}\right) .
$$

Here $I: H_{c}^{n}(F, \mathbb{C}) \times H^{n}(F, \mathbb{C}) \rightarrow \mathbb{C}$ denotes the hermitian Poincaré duality defined by $I(a, b)=\frac{1}{(2 i \pi)^{n}} \int_{F} a \wedge \bar{b}$ and $\rho$ is in $C_{c}^{\infty}\left(X_{\mathbb{R}}\right)$ with $\rho \equiv 1$ near 0 . We use here the notation $i \pi \int_{A} f^{\lambda} \frac{d f}{f} \wedge \square$ for the $\mathbb{R}^{*}$-Mellin transform of the function defined on $\mathbb{R}^{*}$ by $\varphi(s)=\int_{f(s) \cap A} \square$ where $\square$ is a semi-meromorphic $n$-form on $X_{\mathbb{R}}$ with compact support $\left.{ }^{*}\right)$ and poles in $\left\{f_{\mathbb{R}}=0\right\}$.

Recall that the $\mathbb{R}^{*}$-Mellin transform of $\varphi$ is given (see [B99]) by definition by

$$
i \pi M \varphi(\lambda):=\int_{0}^{+\infty} x^{\lambda-1} \varphi(x) d x-e^{-i \pi \lambda} \int_{0}^{+\infty} x^{\lambda-1} \varphi(-x) d x .
$$

The purpose of this article is to give a topological construction of a compact $n$-cycle whose class in $H_{c}^{n}(F, \mathbb{C})_{1}$ is $\gamma(A)$. This complete the results of the paragraph 6 in $[\mathrm{BM}]$. In fact it appears from our proof and $[\mathrm{BM}]$ results that the class of our cycle $\Gamma(A)$ in $H_{c}^{n}(F, \mathbb{C})$ controls completely the poles of $\int_{A} f^{\lambda} \square$ for the given $A$. So the same holds for the asymptotics when $\tau \rightarrow \pm \infty$ of the oscillating integrals $\int_{A} e^{i \tau f} \square$ where $\square$ denotes a $C^{\infty}$-compactly supported $(n+1)$-form on $X_{\mathbb{R}}$.

So to prove existence of a pole in our context it is enough (but also necessary) to prove that the class of $\Gamma(A)$ in $H_{n}(F, \mathbb{C}) \simeq H_{c}^{n}(F, \mathbb{C})$ is not zero. This gives some new light on Jeddi's proof of Palamodov's conjecture (see $[\mathrm{J}])$.

This article was written during a stay in Nagoya graduate School of Mathematics and I want to thank this Institute for its very nice hospitality.

\section{$\S 1$. Some more notations}

We continue with the hypothesis and notations introduced in paragraph 0 (still assuming that an orientation is given on $X_{\mathbb{R}}^{*}$ ).

We shall fix a Milnor representative of $f$, denoted by $f: X_{\mathbb{C}} \rightarrow D_{\delta}$ by choosing a real embedding $X_{\mathbb{R}} \hookrightarrow \mathbb{R}^{N}$ (so $X_{\mathbb{C}} \hookrightarrow \mathbb{C}^{N}$ and $X_{\mathbb{C}} \cap \mathbb{R}^{N}=X_{\mathbb{R}}$ ) and choosing $0<\varepsilon \ll 1$ and $0<\delta \ll \varepsilon$ such that $X_{\mathbb{C}}:=B(0, \varepsilon) \cap f^{-1}\left(D_{\delta}\right)$; we fix a base point $s_{0} \in D_{\delta} \cap \mathbb{R}^{+*}$ and define the Milnor fiber of $f$ to be $f^{-1}\left(s_{0}\right)=F$.

${ }^{(*)}$ Remark that $f$-proper support is enough to define the polar parts of $\int_{A} f^{\lambda} \frac{d f}{f} \wedge \square$. 
Recall now that, given $A$ in $H^{0}\left(X_{\mathbb{R}}-\left\{f_{\mathbb{R}}=0\right\}, \mathbb{C}\right.$ ) (we shall use here the obvious restriction map from $H^{0}\left(X_{\mathbb{R}}^{*}, \mathbb{C}\right)$ to $\left.H^{0}\left(X_{\mathbb{R}}-f_{\mathbb{R}}^{-1}(0), \mathbb{C}\right)\right)$, we have defined in $[\mathrm{BM}]$ the closed $n$ cycles $\delta(A)^{+}:=A \cap f_{\mathbb{R}}^{-1}\left(s_{0}\right)$, oriented as the boundary of the (oriented) open set $A^{+} \cap\left\{f_{\mathbb{R}}<s_{0}\right\}$ and $\delta(A)^{-}:=A \cap$ $f_{\mathbb{R}}^{-1}\left(-s_{0}\right)$ oriented as the boundary of the open set $A^{-} \cap\left\{-s_{0}<f_{\mathbb{R}}\right\}$, where $A=A^{+}+A^{-}$is the decomposition of the sum $A=\sum_{\alpha} a_{\alpha} A_{\alpha}$ according to the sign of $f_{\mathbb{R}}$ on each connected component $A_{\alpha}$ of $X_{\mathbb{R}}-f_{\mathbb{R}}^{-1}(0)$.

Now, using a $C^{\infty}$ trivialization of Milnor's fibration along the half-circle $\left\{s_{0} e^{i \theta}, \theta \in[-\pi, 0]\right\}$ we define $M^{1 / 2} \delta(A)^{-}$as the closed oriented $n$-cycle in $F$ obtained from $\delta(A)^{-}$by direct image along the projection on $F$ given by this trivialisation.

Then we set $\delta(A):=\delta(A)^{+}-M^{1 / 2} \delta(A)^{-}$and denote by $[\delta(A)]$ the classe defined by $\delta(A)$ in $H^{n}(F, \mathbb{C})$. To be precise, the class $[\delta(A)]$ is defined via the hermitian duality $I$ by the formula

$$
I([a], \overline{\delta(A)}):=\underset{(2 i \pi)^{n}}{\perp} \int_{\delta(A)} a
$$

where $a$ is a compactly supported closed $C^{\infty} n$-form on $F$ defining the class $[a]$ in $H_{c}^{n}(F, \mathbb{C})$.

§2. Construction of $\Gamma(A)$ in $H_{n}(F, \mathbb{C}) \simeq H_{c}^{n}(F, \mathbb{C})$

We fix $\varepsilon^{\prime}<\varepsilon^{\prime \prime}<\varepsilon$ with $\varepsilon-\varepsilon^{\prime} \ll \varepsilon$ and define

$$
\begin{aligned}
X & :=B(0, \varepsilon) \cap f^{-1}\left(D_{\delta}\right) \\
X^{\prime} & :=B\left(0, \varepsilon^{\prime}\right) \cap f^{-1}\left(D_{\delta}\right) \\
X^{\prime \prime} & :=B\left(0, \varepsilon^{\prime \prime}\right) \cap f^{-1}\left(D_{\delta}\right)
\end{aligned}
$$

and then $\partial A:=A \cap \partial X^{\prime \prime}$ for our given non zero $A$ in $H^{n}\left(X_{\mathbb{R}}^{*}, \mathbb{C}\right)$. The orientation of this compact $n$-cycle in $X_{\mathbb{R}}^{*}$ is given as the boundary of the open set $A \cap X^{\prime \prime}$. As a compact $n$-chain $\partial A$ has three pieces:

$$
\partial A=\left(\delta\left(A^{-}\right) \cap \bar{X}^{\prime \prime}\right) \cup\left(\delta(A)^{+} \cap \bar{X}^{\prime \prime}\right) \cup \Delta
$$

where the two "vertical" pieces $\delta(A)^{ \pm} \cap \bar{X}^{\prime \prime}$ are obtained by cutting $\delta(A)^{ \pm}$ by $\overline{B\left(0, \varepsilon^{\prime \prime}\right)}$, and where the compact $n$-chain $\Delta$ lies in $X-\bar{X}^{\prime}$ is fibered by $f$ over $\left[-s_{0}, s_{0}\right]$ as a family of compact $(n-1)$-cycles which gives an homology in $X-\bar{X}^{\prime}$ between $\delta(A)^{-} \cap \partial B\left(0, \varepsilon^{\prime \prime}\right)$ and $\delta(A)^{+} \cap \partial B\left(0, \varepsilon^{\prime \prime}\right)$.

The proof of our theorem will follow precisely a move from this compact $n$-cycle $\partial A$ to a compact $n$-cycle $\Gamma(A)$ contained in $F$. To move $\partial A$ to $\Gamma(A)$, 
first fix a $C^{\infty}$ trivialisation of Milnor's fibration over the punctured half disc $\bar{D}_{s_{0}}^{-}-\{0\}=\left\{s \in \mathbb{C} / \operatorname{Im} s \leq 0,|s| \leq s_{0}, s \neq 0\right\}$ which induces the previously fixed trivialisation on the half-circle $\left\{s_{0} e^{i \theta}, \theta \in[-\pi, 0]\right\}$ used to define $M^{1 / 2} \delta(A)^{-}$. We shall also fix a $C^{\infty}$ trivialisation of $f \mid X-\overline{X \cap B\left(0, \varepsilon^{\prime}\right)} \rightarrow$ $D_{\delta}$ which corresponds to a commutative diagram

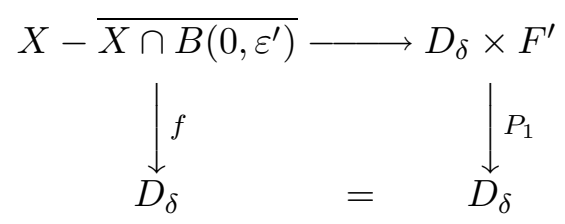

where $F^{\prime}:=F \cap\left(X-\overline{X \cap B\left(0, \varepsilon^{\prime}\right)}\right)$, also compatible with the previous trivialisation.

First we begin by moving $\Delta$ to $\Delta_{0}$ using the above trivialisation (recall that $\left.\Delta \subset X-\overline{X \cap B\left(0, \varepsilon^{\prime}\right)}\right)$ without moving its boundary part, so that we get

$\Delta$

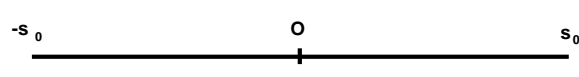

$\Delta_{0}$

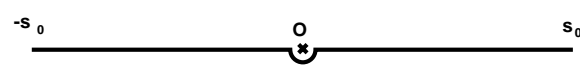

Then we move the compact $n$-cycle $\partial A_{0}:=\partial A-\Delta+\Delta_{0} \subset$ $f^{-1}\left(\bar{D}_{s_{0}}^{-}-\{0\}\right)$ using the above trivialisation of $f$ over this set, so that the vertical part $\delta(A)^{-} \cap \bar{X}^{\prime \prime}$ will follow the half-circle $\left\{s_{0} e^{i \theta}, \theta \in[-\pi, 0]\right\}$, the vertical part $\delta(A)^{+} \cap \bar{X}^{\prime \prime}$ will be fixed, and the $\Delta_{0}$ part moves, using the trivialisation of $f$ on $X-\overline{X \cap B\left(0, \varepsilon^{\prime}\right)}$ from the path $\gamma_{0}$ to the constant path $\gamma_{1}$ equal to $\left\{s_{0}\right\}$ as follows

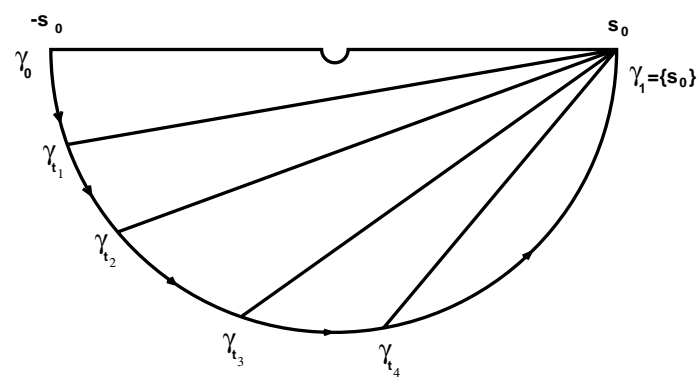


Let us call $\left(\Delta_{t}\right)_{t \in[0,1]}$ this deformation. We shall denote by $\left(\partial A_{t}\right)_{t \in[0,1]}$ the family of compact $n$-cycles in $f^{-1}\left(\bar{D}_{s_{0}}^{-}-\{0\}\right)$ defined for $t \in[0,1]$ by

$$
(\partial A)_{t}:=-\widetilde{\delta}(A)_{s_{0} e^{-i \pi(1-t)}}^{-}+\widetilde{\delta}(A)^{+}+\Delta_{t}
$$

where $\widetilde{\delta}(A)^{ \pm}$is $\delta(A)^{ \pm} \cap \bar{X}^{\prime \prime}$ and where $\widetilde{\delta}(A)_{s_{0} e^{i \theta}}^{-}$is obtained by following the compact $\widetilde{\delta}(A)^{-}$in the above trivialisation along the half-circle.

So we define now the compact oriented $n$-cycle

$$
\Gamma(A):=(\partial A)_{1} \subset F .
$$

By definition we have

$$
\Gamma(A)=\widetilde{\delta}(A)^{+}-M^{1 / 2} \widetilde{\delta}(A)^{-}+\Delta_{1}
$$

where $\Delta_{1}$ is a compact $n$-chain in $F^{\prime}$ so that $\partial \Gamma(A)=\emptyset$.

Remark that this already shows that we have

$$
\operatorname{can}[\Gamma(A)]=[\delta(A)] \quad \text { in } H^{n}(F, \mathbb{C})
$$

because our initial chain $\Delta$ was the boundary in $X-X \cap \overline{B\left(0, \varepsilon^{\prime}\right)}$ of the closed $(n+1)$-chain

$$
\left(A-A \cap B\left(0, \varepsilon^{\prime \prime}\right)\right) \cap f^{-1}\left(\bar{D}_{s_{0}}\right)
$$

(and $\Delta_{0}$ similarily).

As we know that $\partial\left(\widetilde{\delta}(A)^{+}\right)$and $\partial\left(\widetilde{\delta}(A)^{-}\right)$are homologuous in $X-$ $\overline{X \cap B\left(0, \varepsilon^{\prime}\right)}$ as $(n-1)$-compact cycles, for any choice of a compact $n$-chain $\Delta_{2}$ in $F^{\prime}$ such that $\widetilde{\delta}(A)^{+}-M^{1 / 2} \widetilde{\delta}(A)^{-}+\Delta_{2}$ is a compact $n$-cycle in $F$ ( $M^{1 / 2}$ preserves the homology between boundaries), we obtain a compact $n$-cycle in $F$ whose image by

$$
\text { can }: H_{n}(F, \mathbb{C}) \simeq H_{c}^{n}(F, \mathbb{C}) \longrightarrow H^{n}(F, \mathbb{C})
$$

is the class $[\delta(A)]$. But the choice of $\Delta_{2}$ is defined up to a compact $n$-cycle of $F^{\prime}$. As $H_{n}\left(F^{\prime}\right) \simeq H_{n}(\partial F) \simeq H^{n-1}(\partial F)$ is exactly the kernel of can (via the exact sequence $0 \rightarrow$ Ker can $\simeq H^{n-1}(\partial F) \rightarrow H_{c}^{n}(F) \stackrel{\text { can }}{\longrightarrow} H^{n}(F)$ ) to make this construction is just to lift $[\delta(A)]$ to $H_{c}^{n}(A)$, and this is possible by $[\mathrm{BM}]$.

What we have done in the construction of $\Gamma(A)$ is to make a precise choice of $\Delta_{2} \subset F^{\prime}$ by using the component $A$ again.

The following theorem shows that our choice is the good one. 
THEOREM. The cycle $\Gamma(A)$ constructed above satifies the following property:

For any $e \in H^{n}(F, \mathbb{C})_{1}$ we have

$$
I(e, \overline{\Gamma(A)})=(2 i \pi)^{-n} \operatorname{Res}\left(\lambda=0, \int_{A} f^{\lambda} w_{k} \wedge \frac{d f}{f}\right)
$$

where $w_{1}, \ldots, w_{k}$ are semi-meromorphic $n$-forms representing e (i.e. satisfying the condition (A) of paragraph 0$)$.

Moreover we have $\operatorname{can}([\Gamma(A)])=[\delta(A)]$ so, using $[\mathrm{BM}]$, we deduce that $[\Gamma(A)]$ satisfies also:

For any $e \in H^{n}(F, \mathbb{C})_{1}$ represented by $w_{1}, \ldots, w_{k}$

$$
h\left(e, \operatorname{can}\left(\overline{\Gamma(A)}_{1}\right)\right)=(2 i \pi)^{n} P_{2}\left(\lambda=0, \int_{A} f^{\lambda} w_{k} \frac{d f}{f}\right)
$$

where $P_{2}(\lambda=0, F(\lambda))$ is the coefficient of $1 / \lambda^{2}$ of the Laurent expansion of the meromorphic function $f$ at 0 , and where

$$
h: H^{n}(F, \mathbb{C})_{1} \times H^{n}(F, \mathbb{C})_{1} \longrightarrow \mathbb{C}
$$

is the canonical hermitian form defined in $[\mathrm{BM}]$ in our context.

For any $e \in H^{n}(F, \mathbb{C})_{e^{-2 i \pi r}}, 0<r<1$, represented by $w_{1}, \ldots, w_{k}^{(\dagger)}$ we have

$$
I(e, \overline{\Gamma(A)})=\frac{s_{0}^{r}}{(2 i \pi)^{n}} \operatorname{Res}\left(\lambda=-r, \int_{A} f^{\lambda} w_{k} \wedge \frac{d f}{f}\right) .
$$

As an easy consequence we obtain the following corollary, which completes results of $[\mathrm{BM}]$ paragraph 6 .

Corollary.

1) we have $[\Gamma(A)]_{1}=\gamma(A)$

2) $\int_{A} f^{\lambda} \square$ has no poles iff $[\Gamma(A)]=0$ in $H_{n}(F, \mathbb{C})$.

Proof of the theorem. In order to follow easily the moving cycle $(\partial A)_{t}$ and integral on it, it is convenient to introduce a $d$-closed $n$-form $W$ associated to $e \in H^{n}(F, \mathbb{C})_{1}$. Let us fix the logarithm function on $D_{\delta}-D_{\delta} \cap i \mathbb{R}^{+}$ such that the argument is in $]-3 \pi / 2, \pi / 2[$. Now define

$$
W:=\sum_{j=0}^{k-1}(-1)^{j} w_{j} \frac{(\log f)^{j}}{j !}
$$

$(\dagger)$ in this case we have replaced $(A)$ of paragraph 0 by $d w_{j}=r \frac{d f}{f} \wedge w_{j}+\frac{d f}{f} \wedge w_{j-1}$ $\forall j \in[1, k]\left(w_{0}=0\right)$ and $\left[w_{k} / F\right]=e$ 
on the open set $f^{-1}\left(D_{\delta}-D_{\delta} \cap i \mathbb{R}^{+}\right)$of $X$, where $w_{1}, \ldots, w_{k}$ are semimeromorphic $n$-forms on $X$ satisfying $(A)$ of paragraph 0 .

Then we have $d W=0$ and also

$$
\left[\left.W\right|_{F}\right]=\sum_{k-1}^{j=0}(-1)^{j} \frac{\left(\log s_{0}\right)^{j}}{j !} e_{k-j}
$$

where $e_{k-j}:=\left[\left.w_{j}\right|_{F}\right]$ for $j \in[0, k-1]$ in $H^{n}(F, \mathbb{C})$. So $e_{0}=e$.

So we have

$$
\int_{(\partial A)_{0}} W=\int_{\Gamma(A)} W=\sum_{j=0}^{k-1}(-1)^{j} \frac{\left(\log s_{0}\right)^{j}}{j !}(2 i \pi)^{n} I\left(e_{k-j}, \overline{\Gamma(A)}\right) .
$$

Now we have to go from $(\partial A)_{0}$ to $\partial A$.

We define $\int_{\partial A} W$ as follows:

$$
\int_{\partial A} W:=\operatorname{Pf}\left(\lambda=0, \int_{\partial A} f^{\lambda} W\right) .
$$

And we shall precise later on why $\int_{\partial A} W=\int_{(\partial A)_{0}} W$. But thanks to Stokes formula (for $\operatorname{Re} \lambda \gg 0$ ) and analytic continuation we get

$$
\int_{\partial A} W=\operatorname{Res}\left(\lambda=0, \int_{A \cap \bar{X}^{\prime \prime}} f^{\lambda} \frac{d f}{f} \wedge W\right) .
$$

So, modulo our Lemma 2 which will allow us to replace the integration on $A \cap \bar{X}^{\prime \prime}$ by a smooth cut off function $\rho \in C_{c}^{\infty}\left(X_{\mathbb{R}}\right), \rho \equiv 1$ near $\bar{X}^{\prime \prime}$, without changing the polar parts, we obtain, by the definition of $\gamma(A) \in H_{c}^{n}(F, \mathbb{C})_{1}$ recalled in paragraph 0

$$
\sum_{j=0}^{k-1}(-1)^{j} \frac{\left(\log s_{0}\right)^{j}}{j !} I\left(e_{k-j}, \overline{\gamma(A)}\right)=\sum_{j=0}^{k-1}(-1)^{j} \frac{\left(\log s_{0}\right)^{j}}{j !} I\left(e_{k-j}, \overline{\Gamma(A)}\right) .
$$

Now we can conclude easily because we already know from $[\mathrm{BM}]$ that $\operatorname{can}[\gamma(A)]=\operatorname{can}[\Gamma(A)]_{1}$. So for $e_{1}, \ldots, e_{k-1}$ which are in Imcan (because $\operatorname{Im}(T-1) \subset \operatorname{Im}$ can $)$ we know that $I\left(e_{k-j}, \overline{\gamma(A)}\right)=I\left(e_{k-j}, \overline{\Gamma(A)}\right)$ for $j \in[1, k-1]$. So we conclude that

$$
I\left(e_{k}, \overline{\gamma(A)}\right)=I\left(e_{k}, \overline{\Gamma(A)}\right)
$$

and we obtain $[\gamma(A)]=[\Gamma(A)]_{1}$ in $H_{n}(F, \mathbb{C})_{1}$. 
To pass from $\Delta$ to $\Delta_{0}$ we first remark that now we are considering a compact $n$-chain (with fixed boundary) in $X-\overline{X \cap B\left(0, \varepsilon^{\prime}\right)}$ where no singularity occurs for $X_{\mathbb{R}}$ or $f_{\mathbb{R}}$. So locally we can assume that $A=\mathbb{R}^{n+1}$ and $f=x_{0}$ is the first coordinnate. Now let us define a push down of $W$ on $\mathbb{C}$ : via our fixed $C^{\infty}$ trivialisation of $X-\overline{X \cap B\left(0, \varepsilon^{\prime}\right)} \rightarrow D_{\delta}$ we can consider $\Delta$ near $f_{\mathbb{R}}^{-1}(0)$ as a family $\left(\delta_{t}\right)_{t \in[-\eta, \eta]}$ of compact $(n-1)$-cycles in $F^{\prime}$ which are smooth. Let us then consider the submanifold $\nabla$ defined near $\delta_{0}$ as the union of all $\left(t+i \tau, \delta_{t}\right)$ for $\tau \in[-\xi, \xi]$ and $t$ near 0 . So, in fact, we just translate $\Delta$ near 0 along the imaginary axis in our trivialisation compatible with $f$.

Now $\nabla$ is a piece of smooth $(n+1)$-submanifold containing $\Delta$ and with a proper smooth fibration $\left.f\right|_{\nabla}: \nabla \rightarrow \mathbb{C}$ near 0 in $\mathbb{C}$.

Define $\alpha:=(f \mid \nabla)_{*}(W \mid \nabla)$. Then $\alpha$ is a semi-meromorphic $n$-form near 0 in $\mathbb{C}$ which is $d$-closed because $W$ is semi-meromorphic and $d$-closed. Now the following lemma with allow us to pass from $\int_{\partial A} W$ to $\int_{(\partial A)_{0}} W$ :

LEMMA 1. Let $\eta>0$ and denote by $\alpha$ a d-closed semi-meromorphic 1 -form (with pole at $s=0$ ) in a neighbourhood of $\overline{D(0, \eta)}$ in $\mathbb{C}$.

Then we have

$$
\operatorname{Pf}\left(\int_{0}^{\eta} s^{\lambda} i_{+}^{*}(\alpha)-e^{-i \pi \lambda} \int_{0}^{\eta} s^{\lambda} i_{-}^{*}(\alpha)\right)=\int_{-\pi}^{0} j^{*}(\alpha)
$$

where $i_{+}:[0, \eta] \rightarrow \overline{D(0, \eta)}$ and $i_{-}:[-\eta, 0] \rightarrow \overline{D(0, \eta)}$ are the obvious inclusion and where $j$ is given by $j:[-\pi, 0] \rightarrow \overline{D(0, \eta)}, j(\theta)=\eta e^{i \theta}$.

Proof. After reduction to the case $\alpha=d s / s^{k}$ this is an elementary exercice.

To finish the proof of our theorem, it is enough to prove our second lemma:

Lemma 2. Let $\rho \in C_{c}^{\infty}\left(X_{\mathbb{R}}\right)$ with $\rho \equiv 1$ near $\bar{X}^{\prime \prime}$ and let $w$ be a semimeromorphic $n$-form on $X-\overline{X \cap B\left(0, \varepsilon^{\prime}\right)}$ with poles in $f=0$.

Then for any $k \in \mathbb{N}$ the meromorphic function

$$
\lambda \longrightarrow \int_{\left(X-\bar{X}^{\prime \prime}\right) \cap A} f^{\lambda}(\log f)^{k} \rho \frac{d f}{f} \wedge w
$$

has no pole. 
Proof. Of course we have here our previous choice of logarithm. Our assertion is local on $\left(X-\bar{X}^{\prime \prime}\right) \cap \operatorname{Supp} \rho$ so we can assume again that $A=\mathbb{R}^{n+1}$ and that $f_{\mathbb{R}}=x_{0}$ is the first coordinnate.

Far from $\left\{f_{\mathbb{R}}=0\right\}$ there is nothing to prove (and this is the case along the vertical boundary parts of $\bar{X}^{\prime \prime}$ for instance, where $f_{\mathbb{R}}= \pm s_{0}$ ).

Far from $\partial B\left(0, \varepsilon^{\prime}\right)$ (i.e. far from $\Delta$ ) we are reduced to the case of

(*) $\quad \int_{0}^{+\infty} x_{0}^{\lambda-j}\left(\log x_{0}\right)^{k} \sigma\left(x_{0}\right) \frac{d x_{0}}{x_{0}}$

$$
-e^{-i \pi \lambda} \int_{0}^{+\infty}(-1)^{j} x^{\lambda-j}\left(\log x_{0}-i \pi\right)^{k} \sigma\left(-x_{0}\right) \frac{d x_{0}}{x_{0}}
$$

where $\sigma \in C_{c}^{\infty}(\mathbb{R})$ is obtained by integrating first in $x_{1}, \ldots, x_{n}\left(x_{0}^{-j}\right.$ comes from the poles of the semi-meromorphic form $\rho w)$.

Near $\partial B\left(0, \varepsilon^{\prime \prime}\right)$ we are reduced to the same situation but $\sigma \in \mathbb{C}_{c}^{\infty}(\mathbb{R})$ is now obtained by integration of $w$ along $x_{1} \geq 0, x_{2}, \ldots, x_{n}$ where we assume the coordinnates chosen in such a way that $X^{\prime \prime}$ is locally defined by $x_{1}<0$.

To treat $(*)$, use a Taylor expansion of $\sigma$ at $x_{0}=0$ to reduce to the case of

$$
\int_{0}^{\eta} x^{\lambda-j}(\log x)^{k} \frac{d x}{x}-e^{-i \pi \lambda} \int_{0}^{\eta}(-1)^{j} x^{\lambda-j}(\log x-i \pi)^{k} \frac{d x}{x}
$$

which is given, thanks to Cauchy's theorem, by the integral over the half circle $\left\{z=\eta e^{i \theta}, \theta \in[-\pi, 0]\right\}$

$$
\int z^{\lambda-j}(\log z)^{k} \frac{d z}{z}
$$

But this is clearly an entire function of $\lambda$.

\section{REFERENCES}

[B99] D. Barlet, Multiple poles at negative integers for $\int_{A} f^{\lambda} \square$ in the case of an almost isolated singularity, Publ. Res. Inst. Math. Sci., 35 (1999), 571-584.

[BM] D. Barlet and H. M. Maire, Non trivial simple poles at negative integers and mass concentration at singularity, Math. Ann., 323 (2002), 547-587.

[J] A. Jeddi, Preuve d'une conjecture de Palamodov, Topology, 41 (2002), 271-306.

$[\mathrm{P}] \quad$ V. P. Palamodov, Asymptotic expansions of integrals on complex and real regions, Math USSR, 55 (1986), 201-236. 
Universit Henri Poincaré et Institut Universitaire de France Institut Elie Cartan UMR 7502 UHP-CNRS-INRIA

BP $239-F-54506$

Vandoeuvre-lès-Nancy Cedex

France 